Hans Ruddy Angulo Camacho Juan Jose Camacho Camata

Correspondencia a:

Centro de investigación medicina UNITEPC. CIMU

Cochabamba - Bolivia

hans_ruddy@unitepc-mail. com

juan_jcamacho@unitepc-mail.com

Procedencia y arbitraje: No comisionado, sometido a arbitraje externo

Recibido para publicación: 1 de julio del 2018 Aceptado para publicación: 21 de septiembre 2018

\section{UN CASO DE BALANTIDIASIS HUMANA ASINTOMÁTICA}

\section{A CASE OF ASYMPTOMATIC HUMAN BALANCTIDIASIS}

\section{UM CASO DE BALANTIDÍASE HUMANA ASSINTOMÁTICA}

\section{Resumen.}

Se presenta un caso de Balantidiasis humana, el hallazgo del parasito fue accidental, se realizó un estudio coproparasitologico a una estudiante en la práctica de parasitología de la carrera de medicina, observándose quistes y trofozoitos de Balantidium Coli.

Esta no presentaba síntomas actuales ni en el pasado, tampoco tiene antecedentes de contactos con cerdos, siendo este el factor de riesgo que se considera más importante para contraer esta parasitosis

Se usó metronidazol como tratamiento, confirmando después la eliminación del parásito.

Palabras clave: Balantidium Coli. Balantidiasis, Asintomático.

\section{Summary.}

A case of human Balantidiasis is presented, the parasite finding was accidental, a coproparasitological study was made to a student in the practice of parasitology of the medical career, observing Balantidium Coli cyst and trophozoites.

This did not present current symptoms or in the past, nor has a history of contacts with pigs, this being the risk factor that is considered most important to contract this parasitosis

Metronidazole was used as treatment, confirming then the elimination of the parasite.

Keywords: Balantidium Coli. Balantidiasis, Asymptomatic.

\section{Resumo}

Um caso de Balantidíase humana é apresentado, o achado parasitário foi acidental, um estudo coproparasitológico foi feito a um estudante na prática de parasitologia da carreira médica, observando os cisto e trofozoítos Balantidium Coli.

Isso não apresentou sintomas atuais ou no passado, nem tem histórico de contatos com suínos, sendo este o fator de risco considerado mais importante para contrair esta parasitose. O metronidazol foi utilizado como tratamento, confirmando a eliminação do parasita.

Palavras chave: Balantidium Coli. Balantidíase, Assintomática.

\section{INTRODUCCIÓN}

Balantidiasis es una enfermedad parasitaria producida por el protozoario ciliado, Balantidium coli. Considerada una zoonosis, cuya incidencia en el hombre es baja y frecuente en los cerdos, por lo general asociada con malas condiciones de salubridad, 
carencia de servicios básicos (agua potable y alcantarillado). ${ }^{1}$

Se ha encontrado una mayor incidencia en zonas donde la crianza de cerdos no tiene un adecuado control sanitario. ${ }^{2}$

Es frecuente en adultos, esto se debe a las actividades laborales como ser criadores y/o faenadores de cerdos. ${ }^{3}$

B. coli habita en el ciego y colon ascendente. Existen tres tipos de presentación: a) portador sano, forma asintomática, b) forma crónica sintomática, caracterizada por diarreas alternando con estreñimiento, y c) forma disentérica o aguda pudiendo dar lugar a cuadros fulminantes .4-7

Es de interés presentar este caso clínico con el fin de contribuir a la bibliografía existente en Cochabamba Bolivia sobre el tema.

\section{CASO CLÍNICO}

Mujer de 19 años procedente Trinidad capital del Beni departamento de Bolivia; estudiante de medicina en Cochabamba.

Ella no presenta ningún síntoma, y se hace un examen coproparasitologico, como parte de la práctica de la materia de parasitología del cuarto semestre gestión l-2017.

\section{Coproparasitologico simple:}

Macroscopía: heces pastosas, marrones, sin mucus, sin pus y sin sangre.

Microscopía: se observa, formas esfericas con pared quística, citoplasma y membrana ciliada por debajo de la pared quística un macronúcleo y otras ovaladas, rodeadas de cilios, con gran movilidad de desplazamiento y de rotación; núcleo grande y con citostoma. Se identifican fácilmente como quistes y trofozoitos de B. coli, (figura 1).

El hallazgo fue accidental, se realizó tratamiento con metronidazol a la dosis de $500 \mathrm{mg}$ cada 8 horas por 7 días; luego para verificar eliminación del parasito se hicieron exámenes coproparasitologicos seriados que fueron negativos.

\section{Figura 1.}

Estudio coproparasitologico en fresco. Abundantes trofozoitos ciliados de $\mathrm{Ba}$ lantidium coli.






\section{DISCUSIÓN.}

La Balantidiasis afecta el intestino grueso del humano. Se alimenta de detritus e hidratos de carbono intraluminales y acumula almidón en su citoplasma. Puede, lesionar las células de la mucosa intestinal llegando a producir úlceras planas múltiples. ${ }^{4}$

El mecanismo de transmisión es fecal oral, siendo los quistes las formas infectantes; éstos se producen cuando los trofozoitos son arrastrados con las heces fecales y a veces se completa el enquistamiento en las deposiciones. ${ }^{5}$

Los humanos adquieren la infección a partir de alimentos (verduras crudas), o cosas contaminados con heces de humanos o cerdos, ya que en los lugares donde crían estos animales las infecciones tienen mayor incidencia. ${ }^{6}$

Aunque las cepas del cerdo son de difícil transmisión al hombre, hecho avalado por estudios epidemiológicos, una vez que se adquieren pueden permanecer y mantenerse por mucho tiempo, así como producir brotes epidémicos. Los alimentos con alto contenido de hidratos de carbono favorecen las infecciones. Otros mamíferos también sirven de reservorio, pero las personas en contacto con cerdos son las más expuestas. $^{7}$

Ya que la ingestión de quistes no lleva necesariamente a la infección, es de suponer que, se necesita un hospedero humano adecuado, con dietas que facilitan la infección o que la carga parasitaria sea grande y por mucho tiempo, para que algunas cepas se adapten al mismo. ${ }^{8}$

Según el estudio realizado por Esteban J. La zona norte del altiplano de Bolivia presenta una alta prevalencia en niños de la cultura Aymara que cursan con formas asintomática, asociadas a deficiencias en las condiciones de saneamiento y al contacto estrecho con animales. Algunos brotes epidémicos se han producido por catástrofes ambientales que determinaron contaminación de los abastecimientos de agua. ${ }^{9}$

Este protozoario es poco frecuente y se lo encuentra en pequeños grupos de la población muy postrada o que tiene relación con la crianza y comercialización de cerdos; desconociendo su prevalencia real en Bolivia. ${ }^{10}$

Desconocemos la fuente de infección de esta estudiante, ya que no tuvo contactos con cerdos, siendo este el factor de riesgo que se considera más importante para contraer esta parasitosis, también indica que no presento ningún síntoma, como ser dolor abdominal y/o episodios de diarrea. No hubo en el entorno familiar de los últimos años ninguna persona con diarrea o con sintomatología sospechable.

Con respecto al tratamiento se indicó metronidazol, por ser un antiparasitario con menos efectos secundarios y casi con la misma efectividad que la tetraciclina. ${ }^{5}$

Hubo una buena respuesta terapéutica, con la eliminación de los trofozoitos, y no fue necesario complementarlo con una dieta estricta basada en derivados lácteos, yogur y con supresión de los hidratos de carbono. ${ }^{11}$

\section{BIBLIOGRAFÍA}

1. Faust, E. C; Russell, P. F., and Jung, R. C. "Parasitología Clínica". Edit. Salvat, 1974.

2. Atias, A., Neghme, A. "Parasitología Clínica". Edit. Intermedia, Buenos Aires, 1979. 
3. Lumbreras, H. "Balantidiasis en el Perú". Rev. Medica peruana. 25: 463-531, 1954.

4. Tálice R.V. Balantidiosis. In: Tálice R.V. Enfermedades parasitarias del hombre. Montevideo: Ed. Científica Sindicato Médico del Uruguay, 1944: 582-600.

5. Osimani JJ. Ciliados. Balantidiasis. In: Osimani JJ. Parasitología Médica. Montevideo: Librería Médica, 1982: 146-55. Tomo I.

6. 6.- García LS. Intestinal Protozoa, Flagellates and Ciliates. In: García LS. Diagnostic Medical Parasitology. 4th ed. Santa Mónica. California: LSG Asociates, 2001: 6-59.

7. Beaver PCh, Jung RC, Cupp EW. Protozoarios ciliados. Balantidium coli. In: Beaver PCh, Jung RC, Cupp EW. Parasitología Clínica. $2^{\mathrm{a}}$ ed. Barcelona: Salvat, 1986: 231-5.

8. Yazar S, Altuntas F, Sahin I, Atambay M. Dysentery caused by Balantidium coli in a patient with non-Hodkin's lymphoma from Turkey. World J Gastroenterol 2004; 10(3): 458-9.

9. Esteban JG, Aguirre C, Angles R, Ash LR, Mas-Comas S. Balantidiasis in Aymara children from the northern Boliv- ian Altiplano. Am J Trop Med Hyg 1998; 59(6): 922-7.

10. Sergio Mollinedo P./ Cecília Prieto B. El Enteroparasitismo en Bolivia (Memoria de la investigación 1975-2004) 2006; 38.

11. Tálice R.V., Nieto C. Primer caso de Balantidiasis humana. An Fac Med Montevideo 1932; 17: 115-9. 